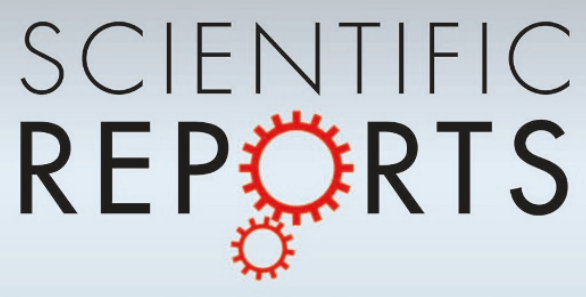

OPEN

SUBJECT AREAS:

ELECTRONIC AND

SPINTRONIC DEVICES

SPINTRONICS

ELECTRONIC PROPERTIES AND MATERIALS

Received

18 September 2014

Accepted

16 January 2015

Published

13 February 2015

Correspondence and requests for materials should be addressed to

Y.-H.Z. lyhzhao@ sicnu.edu.cn) or W.J.

(wii@ruc.edu.cn)

\section{Continuously tunable electronic structure of transition metal dichalcogenides superlattices}

\author{
Yong-Hong Zhao ${ }^{1,2}$, Feng Yang ${ }^{1,3,4}$, Jian Wang ${ }^{2}$, Hong Guo ${ }^{5}$ \& Wei ji ${ }^{3,4}$
}

\begin{abstract}
${ }^{1}$ College of Physics and Electronic Engineering, Institute of Solid State Physics, Sichuan Normal University, Chengdu 610068, China, ${ }^{2}$ Department of Physics and the Center of Theoretical and Computational Physics, The University of Hong Kong, Hong Kong, China, ${ }^{3}$ Department of Physics, Renmin University of China, Beijing 100872, China, ${ }^{4}$ Beijing Key Laboratory of Optoelectronic Functional Materials \& Micro-nano Devices, Renmin University of China, Beijing 100872, China, ${ }^{5}$ Centre for the Physics of Materials and Department of Physics, McGill University, 3600 rue University, Montreal PQ, Canada H3A 2T8.
\end{abstract}

Two dimensional transition metal dichalcogenides have very exciting properties for optoelectronic applications. In this work we theoretically investigate and predict that superlattices comprised of $\mathrm{MoS}_{2}$ and $\mathrm{WSe}_{2}$ multilayers possess continuously tunable electronic structure with direct bandgaps. The tunability is controlled by the thickness ratio of $\mathrm{MoS}_{2}$ versus $\mathrm{WSe}_{2}$ of the superlattice. When this ratio goes from $1: 2$ to $5: 1$, the dominant $\mathrm{K}-\mathrm{K}$ direct bandgap is continuously tuned from $0.14 \mathrm{eV}$ to $0.5 \mathrm{eV}$. The gap stays direct against $-0.6 \%$ to $2 \%$ in-layer strain and up to $-4.3 \%$ normal-layer compressive strain. The valance and conduction bands are spatially separated. These robust properties suggest that $\mathrm{MoS}_{2}$ and $\mathrm{WSe}_{2}$ multilayer superlattice should be a promising material for infrared optoelectronics.

$\mathrm{t}$ has been shown recently that monolayer (ML) transition metal dichalcogenides (TMDC) have very interesting properties as emerging materials for optoelectronic devices ${ }^{1-7}$. These ML-TMDCs have direct bandgaps in the visible frequency range and some of which have strong spin-orbit coupling (SOC). Prototypes of photoactive devices made by these materials already offer a quantum efficiency of up to $30 \%{ }^{8}$ and a theoretical power conversion efficiency up to $1 \%$ in solar cell applications ${ }^{9}$. Interestingly, several TMDCs are indirect bandgap material in the bulk form, but it undergoes an indirect to direct transition when the thickness reduces to ML. These materials include $\mathrm{MoS}_{2}, \mathrm{MoSe}_{2}, \mathrm{WS}_{2}$, and $\mathrm{WSe}_{2}{ }^{10,11}$.

To realize a powerful TMDC optoelectronics, a most striking scenario is to make the bandgap tunable. While strain inside the layers of TMDC can linearly decrease the bandgap of monolayer $\mathrm{MoS}_{2}{ }^{12,13}$, however, it also makes

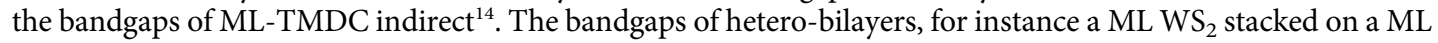
$\mathrm{WSe}_{2}$, are predicted to be direct ${ }^{15,16}$. When the hetero-bilayers periodically repeat to form a superlattice, however, an indirect bandgap again emerges. It appears that ML-TMDC is paramount for retaining the direct bandgap ${ }^{10-21}$. This restriction strongly limits the fundamental building blocks for TMDC optoelectronics. In addition, a significant portion of solar spectrum is in the infrared range. A photovoltaic device that has narrower direct bandgap thus naturally absorbs more energy of sunlight. In light of these, a very important open problem is how to design TMDC systems having both tunable electronic structures and narrower direct bandgaps. It is the purpose of this work to provide a solution to this problem.

\section{Results}

In particular, we report a discovery that extends the candidates of building blocks for TMDC optoelectronic devices from only ML to potentially limitless possibilities. By first principles electronic structure theory we show that TMDC multilayer superlattices offer direct bandgap and continuously tunable electronic structures. The tunability is achieved by the thickness ratio of the multilayers across the hetero-interface. Namely, the $1: 1$

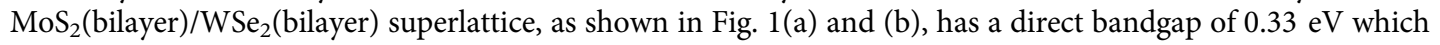
is continuously tunable by changing the $\mathrm{MoS}_{2}$ and $\mathrm{WSe}_{2}$ ratio. The direct bandgap is also robust against in-layer ( $a$ direction) strain from $-0.6 \%$ to $2 \%$, and normal-layer ( $c$ direction) uniaxial strain up to $-4.3 \%$. Moreover, the valance and conduction bands were found spatially separated. These interesting results strongly suggest that TMDC multilayer superlattices may well be the emerging optoelectronic device material in the mid-infrared frequency range. 


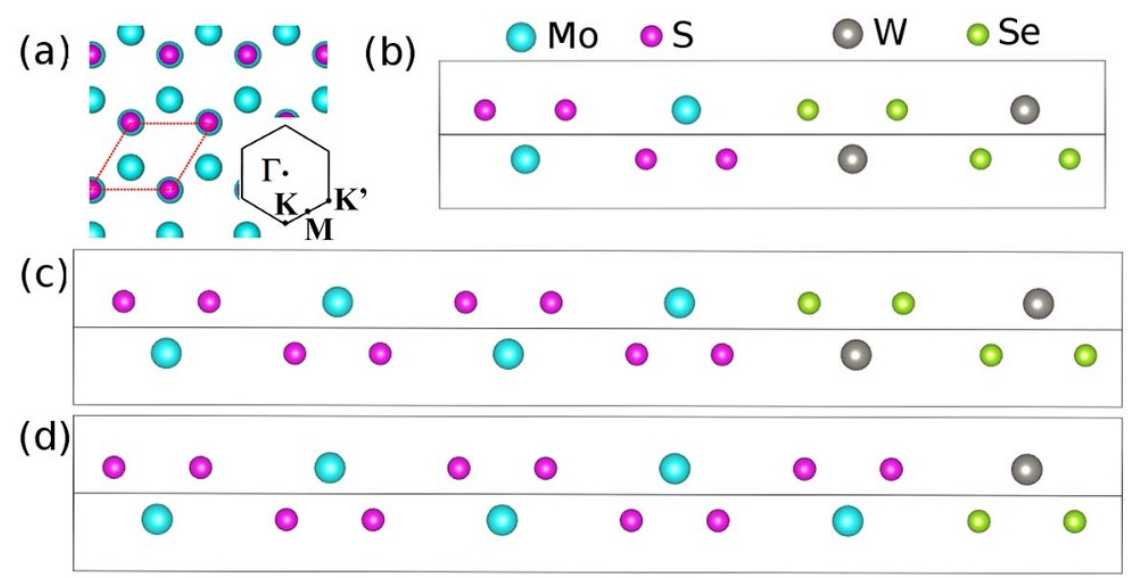

Figure 1 Crystal structure of representative superlattices. (a) top view of a superlattice as indicated by solid lines, and its associated first Brillouin zone; side views of (b) the $1: 1$ superlattice where $\mathrm{MoS}_{2}: \mathrm{WSe}_{2}$ equals to $1: 1$; (c) the $2: 1$ superlattice where $\mathrm{MoS}_{2}: \mathrm{WSe}_{2}$ equals to 2:1; (d) the $5: 1$ monolayer superlattice where all $\mathrm{MoS}_{2}$ and $\mathrm{WSe}_{2}$ bilayers were replaced by the corresponding monolayers.

Besides the $1: 1$ superlattice, two additional families of superlattices were investigated. In the first, the thickness of $\mathrm{WSe}_{2}$ is kept as constant, i.e. a bilayer, and the thickness of $\mathrm{MoS}_{2}$ is varied from bilayer to four layers (see Fig. 1 (c)) and eventually to ten layers. In other words, we increase the $\mathrm{MoS}_{2} / \mathrm{WSe}_{2}$ ratio $\left(\mathrm{N}_{\mathrm{MoS}_{2}}: \mathrm{N}_{\mathrm{WSe}_{2}}\right)$ from $1: 1$ to $5: 1$. In the second family of superlattice, the thickness of $\mathrm{MoS}_{2}$ is kept fixed at bilayer and the ratio decreases from $1: 1$ to $1: 5$. Atomic structures including the volume and shape of the lattice and all internal atomic coordinates were fully optimized for each superlattice and the results were presented in Table 1. The associated in-layer lattice parameter $a$ varies from $3.214 \AA$ to $3.294 \AA$, close to the values of bulk $\mathrm{MoS}_{2}$ and $\mathrm{WSe}_{2}$ respectively, when the $\mathrm{N}_{\mathrm{MoS}_{2}}: \mathrm{N}_{\mathrm{WSe}_{2}}$ ratio decreases from $5: 1$ to $1: 5$.

Electronic properties of layered materials usually have a strong thickness dependence ${ }^{22,23}$. We firstly investigate the evolution of bandgaps as a function of the relative thickness of $\mathrm{MoS}_{2}$ and $\mathrm{WSe}_{2}$, i.e. the $\mathrm{MoS}_{2} / \mathrm{WSe}_{2}$ ratio. The strength of SOC is proportional to $Z^{4}$ where $Z$ is the effective atomic number. Transition metal atoms, like Mo and especially $\mathrm{W}$, have a rather large atomic number, which shall give rise to a sufficiently large spin-orbit interaction and may qualitatively change the electronic structures around the Fermi Level. We, therefore, calculated the bandstructures with the inclusion of the SOC correction from the fully relaxed atomic structures of these superlattices. Figure 2(a) shows the values of the $\mathrm{K}-\mathrm{K}$ direct and $\Gamma-\mathrm{K}$ indirect bandgaps as a function of the $\mathrm{MoS}_{2} / \mathrm{WSe}_{2}$ ratio. Both the direct and indirect gaps becomes larger as a function of this ratio. The largest direct (indirect) gap is $0.50 \mathrm{eV}(0.65 \mathrm{eV})$ found at $\mathrm{N}_{\mathrm{MoS}_{2}}: \mathrm{N}_{\mathrm{WSe}_{2}}=5: 1$; while the smallest direct (indirect) gap reaches $0.14 \mathrm{eV}(0.07 \mathrm{eV})$ when $\mathrm{N}_{\mathrm{MoS}_{2}}: \mathrm{N}_{\mathrm{WSe}_{2}}=1: 5$. We conclude that both the direct and indirect bandgaps are continuously tunable, going from nearly zero-gap to narrow gap semiconductors according to the $\mathrm{MoS}_{2} / \mathrm{WSe}_{2}$ ratio of the superlattice. If all bilayers in the 5:1 superlattice are replaced with monolayers, as shown in Fig. 1(d), the superlattrice retains the direct bandgap of about $0.52 \mathrm{eV}$. The tuning of the bandgaps can be explained by the stacking and SOC induced VB separations. Very briefly, the thinner the $\mathrm{WSe}_{2}$ in the superlattice, the smaller the VB separation at the K point, hence the larger the $\mathrm{K}-\mathrm{K}$ direct bandgap. In the monolayer case shown in Fig. 1(d), the stacking induced VB separation is further suppressed, giving rise to an even larger $\mathrm{K}-\mathrm{K}$ gap. These behaviors will be elucidated further below, and we refer interested readers to Fig. S2(a) of the associated Supplementary Information for more details.

Strain significantly affects bandgaps of TMDC ${ }^{6,14,24,25}$ and usually changes the dominant bandgap of ML TMDC from direct to indirect as discussed above. While for multilayer $\mathrm{WSe}_{2}$, strain can induce a direct bandgap ${ }^{26}$. Our superlattice, however, resists such an undesirable change. As an example, we investigated the strain effect using the $5: 1$ superlattice which has the largest direct and indirect bandgaps among all superlattices we studied. Figures 2(b) and (c) plot the evolution of the bandgap as a function of in-layer and normal-layer strains, respectively. In-layer strain was applied by varying the inlayer lattice constant $a$ and keeping it fixed during structural optimization, which ensures the structure relaxed with the optimized Poisson's ratio. Figure 2(b) plots four bandgaps, the K-K direct bandgap and the $\Gamma-\mathrm{K}, \Gamma-\mathrm{I}$, and $\mathrm{K}-\mathrm{I}$ indirect bandgaps, versus $\Delta a$ from $-3 \%$ to $4 \%$. The $\mathrm{K}-\mathrm{K}$ direct bandgap (red line) is dominant in these superlattices in a range of $-0.6 \% \leq \Delta a \leq 2.0 \%$. Besides this range, the K-I or $\Gamma$-K indirect bandgaps are the smallest bandgaps. Normallayer compressive strain was applied by the same scheme where the normal-layer lattice parameter $c$ was shortened up to $10 \%$ at a step of $2 \%$. Values of K-K direct and $\Gamma-\mathrm{K}$ indirect bandgaps were plotted in Fig. 2(c). The direct bandgap is almost a constant but the indirect gap rapidly decreases when the strain increases up to $10 \%$, resulting in a transition from direct to indirect at a compressive ratio of $\sim-4.3 \%$. This strain corresponds to an external pressure of $\sim 3 \mathrm{GPa}$. We thus conclude that the proposed superlattices are robust against strain as far as the direct gap is concerned.

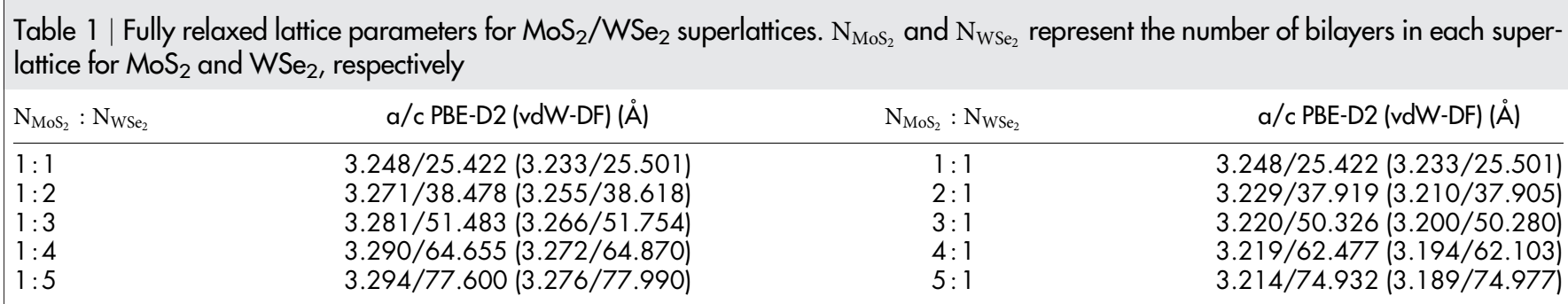



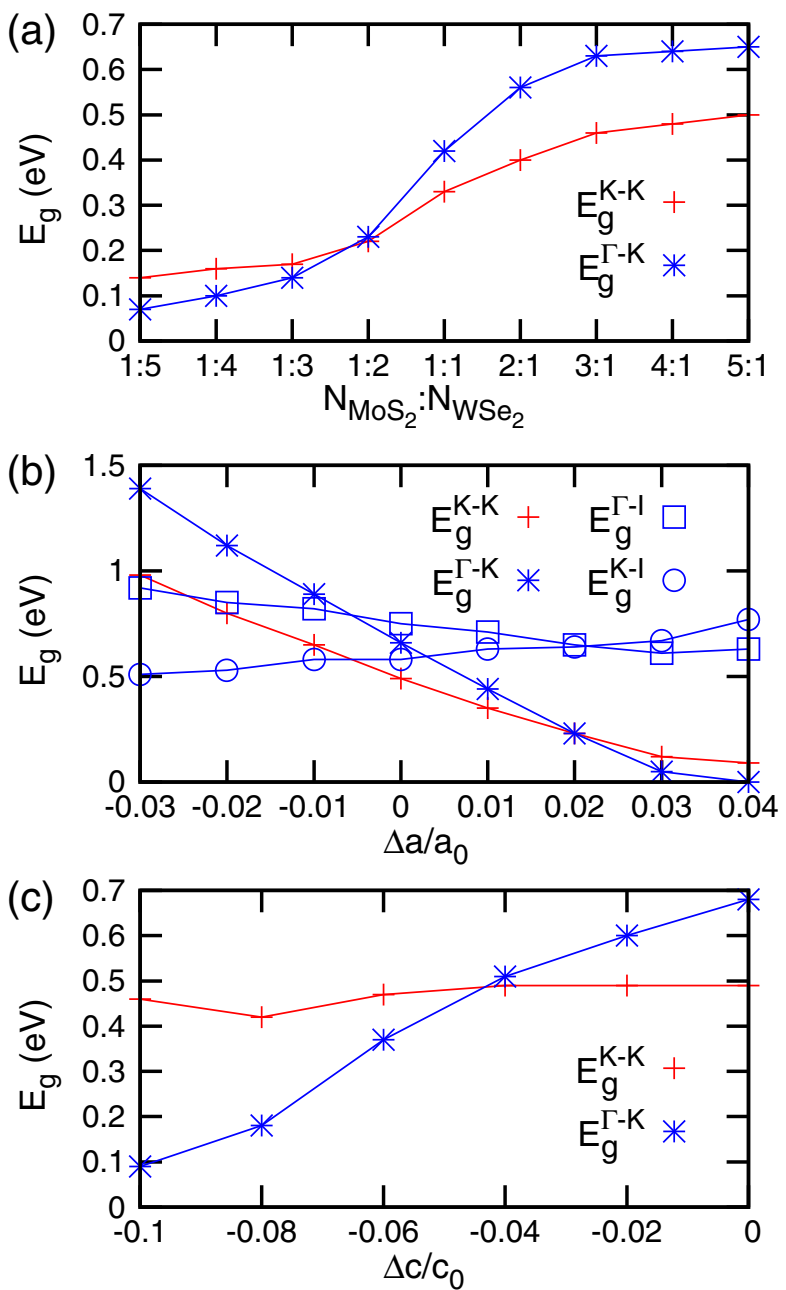

Figure $2 \mid$ Direct (K-K) and indirect ( $\Gamma-\mathrm{K}, \Gamma-\mathrm{I}$, and $\mathrm{K}-\mathrm{I})$ bandgaps of $\mathrm{MoS}_{2} / \mathrm{WSe}_{2}$ superlattice as a function of: (a) the $\mathrm{MoS}_{2}$ and $\mathrm{WSe}_{2}$ ratio; (b) the in-layer strain and (c) the normal-layer compressive strain. The red color and the plus symbols represent $\mathrm{K}-\mathrm{K}$ direct gap; the blue color and the star, square and circle symbols represent the $\Gamma-\mathrm{K}, \Gamma-\mathrm{I}$ and $\mathrm{K}-\mathrm{I}$ indirect gaps, respectively.

\section{Discussion}

Having established the properties of tunable electronic structure and direct gap of the superlattices based on PBE-DFT calculation, we now discuss the origin of the direct bandgap by examining the bulk $\mathrm{MoS}_{2}$, $\mathrm{WSe}_{2}$ and the 1:1 superlattice, as shown in Fig. 3. Band structures based on the HSE $06^{27}$ calculation are given in Fig. 3, in order to avoid the wellknown bandgap underestimation of PBE. Either with or without SOC, the bulk materials have indirect bandgaps between $\Gamma$ and a certain intermediate point (I) along $\Gamma$-K, as shown in Fig. 3(a) and (b), consistent with the previous reports ${ }^{17-20}$. The most remarkable change of the bulk band structure induced by SOC is the significant VB splitting at the $\mathrm{K}$ point of $\mathrm{WSe}_{2}$ (see Fig. 3(b)), though this change is minor for $\mathrm{MoS}_{2}$ (Fig. 3(a)). The non-SOC band structure of the $1: 1$ superlattice (black solid line in Fig. 3(c)) appears to be a combination of the bulk band structures of $\mathrm{MoS}_{2}$ and WSe $\mathrm{W}_{2}$. Wave function visualization (see Figs. 4 and S1) shows that the two highest $\mathrm{VBs}$ (VB1 and VB2) mainly originate from the $\mathrm{VBs}_{\text {of }} \mathrm{WSe}_{2}$; and the two lowest CBs (CB1 and CB2) are solely contributed by the CBs of $\mathrm{MoS}_{2}$. This combination significantly reduces the $\mathrm{K}-\mathrm{K}$ bandgap. The VBM, a mixture of $\mathrm{MoS}_{2}$ and $\mathrm{WSe}_{2}$ states, is found at $\mathrm{K}$, which is $60 \mathrm{meV}$ higher in energy than the VB1 at $\Gamma$. We align the SOC corrected band structure (red dashed line in Fig. 3(c)) to the nonSOC one by VB1 at the $\Gamma$ point in order to better understand the role (a) $\mathrm{MoS}_{2}$

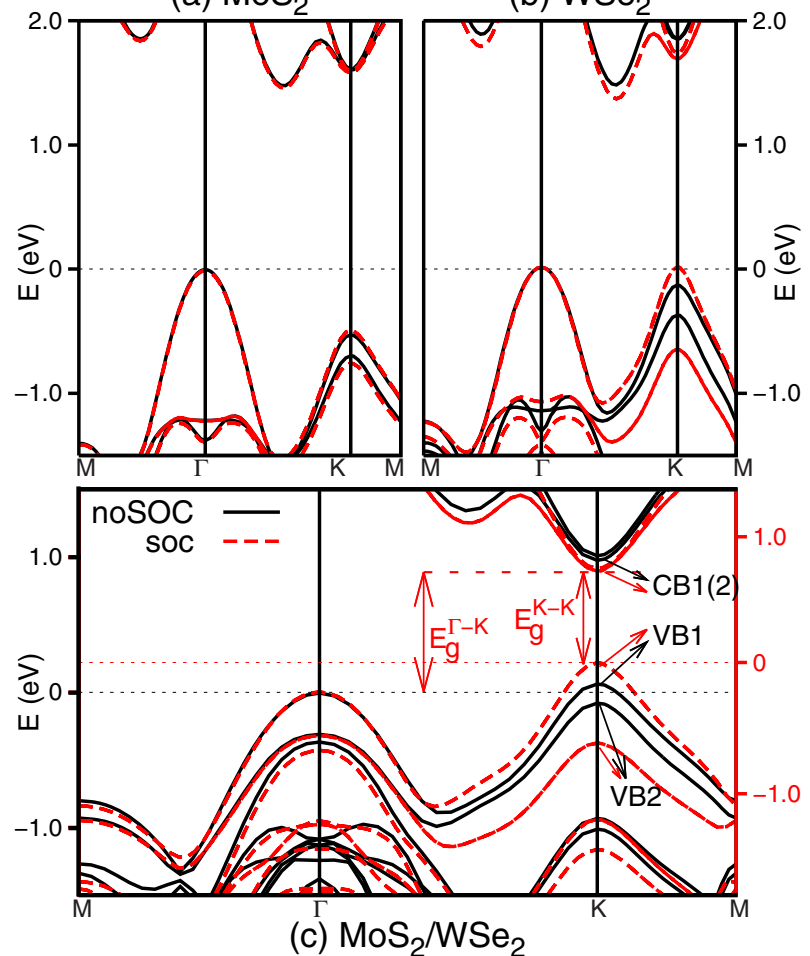

(c) $\mathrm{MoS}_{2} / \mathrm{WSe}_{2}$

(b) $\mathrm{WSe}_{2}$

1.0
Figure 3 Band structures of (a) bulk $\mathrm{MoS}_{2}$ and (b) $\mathrm{WSe}_{2}$, and (c) the $1: 1$ superlattice shown in Fig. 1(a). Black solid and red dashed lines show the results without and with SOC, respectively. Horizontal thin dotted lines mark the energies of VBMs for non-SOC (black) and SOC (red) results. $\mathrm{E}_{g}^{K-K}$ and $\mathrm{E}_{g}^{\Gamma-K}$ represent the direct and indirect gaps of the superlattice, respectively. States $\mathrm{CB} 1, \mathrm{CB} 2, \mathrm{VB} 1$, and $\mathrm{VB} 2$ are the four states around the bandgap.

of SOC. Similar to the $\mathrm{WSe}_{2}$ case, the SOC correction enlarge the gap between VB1 and VB2 and pushed the energy of VB1 higher of about $150 \mathrm{meV}$. Together with the CBM at $\mathrm{K}$, we arrive at a direct bandgap of about $0.68 \mathrm{eV}$ at $\mathrm{K}\left(E_{g}^{K-K}\right)$. This bandgap is also tunable by inlayer and normal-layer strains which change the inter- and intralayer electronic coupling moving upwards or downwards the energy of VB at the K point. In light of this, a superlattice could experience a direct to indirect transition under a certain external strain, as observed in Fig. 2(b) and (c).

The microscopic physics is further revealed by plotting the wave functions of CB1, CB2, VB1, and VB2 at the K point. We denote them as $\Psi_{\mathrm{K}}^{\mathrm{CB} 1}, \Psi_{\mathrm{K}}^{\mathrm{CB} 2}, \Psi_{\mathrm{K}}^{\mathrm{VB} 1}, \Psi_{\mathrm{K}}^{\mathrm{VB} 2}$, and visualize them by plotting their square norm. Figures $4(\mathrm{a})-(\mathrm{f})$ clearly show that $\Psi_{\mathrm{K}}^{\mathrm{CB1}(2)}$ is mainly composed of Mo $d_{z^{2}}$ and S 3-fold $p$ orbitals. Although $\Psi_{\mathrm{K}}^{\mathrm{VB} 1}$ and $\Psi_{\mathrm{K}}^{\mathrm{VB} 2}$ are separated in energy, Figs. 4(g)-(l) explicitly show that they originate from the same combination of inlayer 3 -fold $\mathrm{W} d$ and Se $p$ states. The results without SOC thus indicate that stacking the materials breaks the energetic degeneracy of VB1 and VB2 but keeps the composition of their wave functions. By including SOC, on the other hand, the composition of the wave functions are also broken as shown by panels $(b, c, e, f, h, i, k, l)$ of Fig. 4 . The wave function mainly resides at one side of a bilayer at a certain $\mathrm{K}$ point, for example, most portion of $\Psi_{\mathrm{K}}^{\mathrm{VB} 1}$ locates at the right side of the $\mathrm{MoS}_{2} / \mathrm{WSe}_{2}$ interface, while $\Psi_{\mathrm{K}^{\prime}}^{\mathrm{VB} 1}$ at the left side [Fig. 4(h,i)]. In other words, SOC makes the originally degenerate $\mathrm{K}$ and $\mathrm{K}^{\prime}$ points distinguishable. The enlarged separation moves the VBM from the $\Gamma$ to the $\mathrm{K}$ point, bringing about the direct K-K gap in these superlattices. We conclude that SOC plays a critical role to produce the direct bandgaps in the proposed superlattices. 

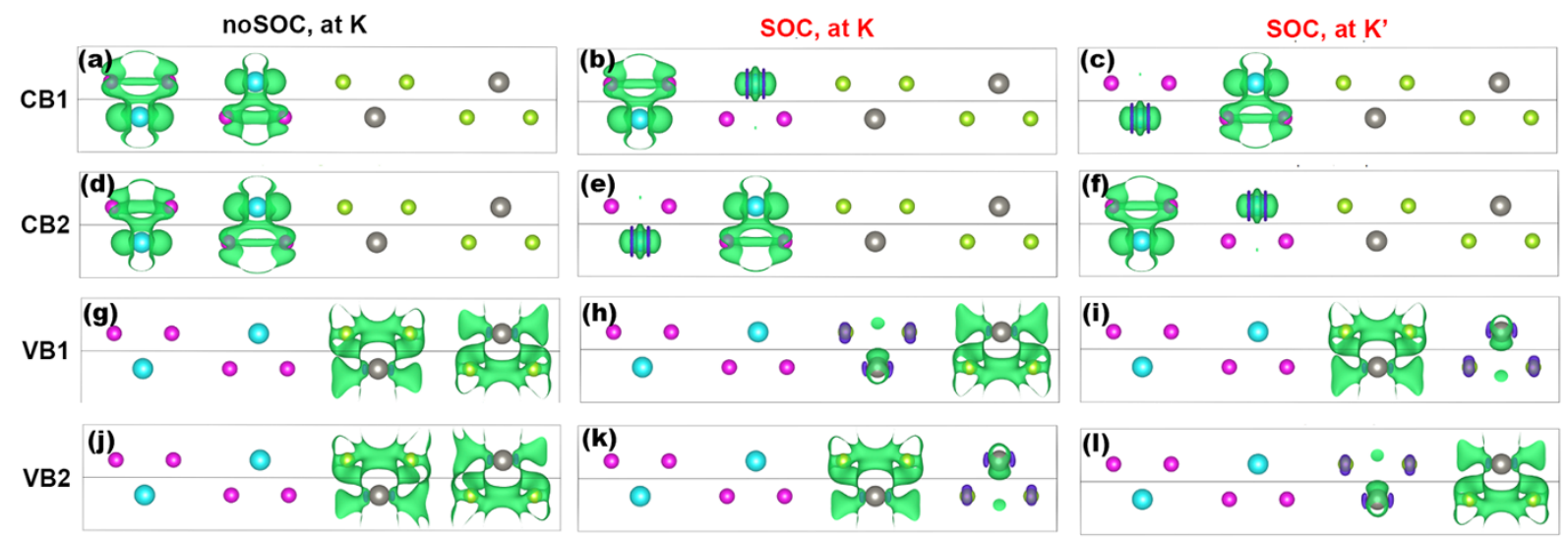

Figure $4 \mid$ Visualized wavefunctions of the $1: 1$ superlattice, for CB1 (a)-(c), CB2 (d)-(f), VB1 (g)-(i) and VB2 (j)-(l), respectively. Non-SOC result at K and SOC results at $\mathrm{K}$ and $\mathrm{K}^{\prime}$ are shown at the left, middle and right panels, respectively. The isosurface is $0.001 e / \AA^{3}$.

Optical absorption spectrum directly reflects the role of SOC. Figure 5 shows the absorption spectra of the $1: 1$ superlattice computed at the PBE level with (blue dashed line) and without (red solid line) inclusion of SOC. The absorption edge of the first peak in the red line start at about $0.5 \mathrm{eV}$, which corresponding to the fundamental bandgap of the superlattice without SOC. After turning on the SOC interaction, this peak definitely splits into two peaks. The lower energy one starts at roughly $0.35 \mathrm{eV}$, corresponding to the bandgap of the superlattice with inclusion of SOC. Moreover, by including SOC interaction, the absorption coefficients become much larger, which is consistent with the expected indirect-direct transition of bandgap at the PBE level. It is worthy to emphasize that the spatially separated valance and conduction bands is a desirable property since it should help to reduce the recombination of electron-hole pairs and thus lead to a process with a higher quantum efficiency, such as a charge transfer induced ultrafast photoelectron generation ${ }^{28}$.

In summary, we propose a novel $\mathrm{MoS}_{2} / \mathrm{WSe}_{2}$ superlattice that offer continuously tunable electronic structures and direct bandgaps which are robust against reasonable ranges of external strains. We identify that the robust direct bandgap is resulted from a strong spinorbit coupling in $\mathrm{WSe}_{2}$ and the band alignment between $\mathrm{MoS}_{2}$ and $\mathrm{WSe}_{2}$. Either the $\mathrm{MoS}_{2} / \mathrm{WSe}_{2}$ thickness ratio or an additional in-layer external stress can continuously change the direct gap from $0.14 \mathrm{eV}$ to $0.50 \mathrm{eV}$. The spatially separated valance (on $\mathrm{WSe}_{2}$ ) and conduction $\left(\right.$ on $\mathrm{MoS}_{2}$ ) bands is, from the application point of view, another attractive property of the proposed superlattice. On the fundamental

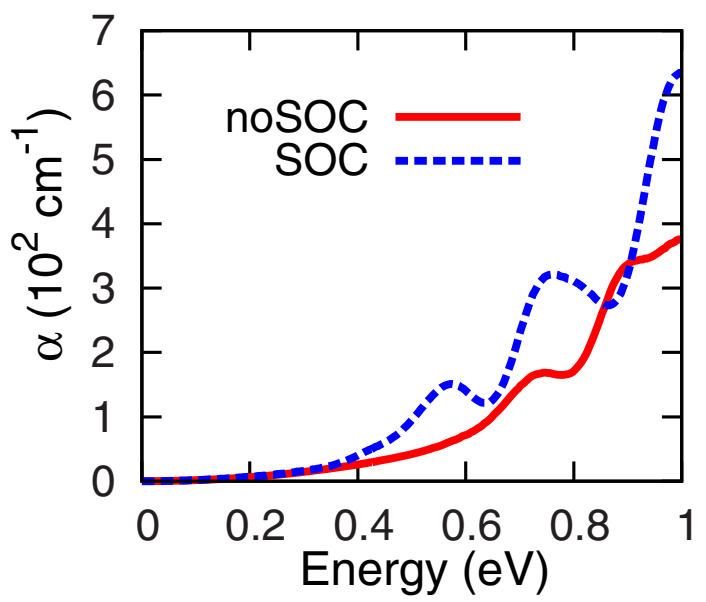

Figure 5 Optical absorption spectra of the $1: 1$ superlattice under PBE approximation. The red line shows that without SOC and the blue line shows the SOC one. side, if valance electrons are excited to the conduction bands by polarized light, the spatially separated VB and CB shall give rise to a magnetic moment normal to the $\mathrm{MoS}_{2} / \mathrm{WSe}_{2}$ interface. Finally, the distinguishable $\mathrm{K}$ and $\mathrm{K}^{\prime}$ points caused by SOC make these superlattices interesting in valley-electronics ${ }^{29-31}$. These properties strongly suggest that the proposed TMDC multilayer-based superlattices are highly promising for optoelectronic and photovoltaic systems in the infrared range, which may promote an new research field for TMDC. Indeed, very recently, a similar superlattice material comprised of PbSe and $\mathrm{MoSe}_{2}$ multilayers was successfully synthesized $^{32}$ and SOC induced VB splitting was experimentally observed ${ }^{33}$.

\section{Methods}

Density functional theory calculations were performed using the generalized gradient approximation for the exchange-correlation potential ${ }^{34}$, the projector augmented wave method ${ }^{35,36}$, and a plane wave basis set as implemented in the Vienna ab-initio simulation package ${ }^{37,38}$. The energy cutoff for plane-wave basis was set to $400 \mathrm{eV}$ for all calculations. A $k$-mesh of $24 \times 24 \times 1$ or $24 \times 24 \times 3$, depending on different values of lattice parameter $c$, was adopted to sample the first Brillouin zone. In geometry optimization, dispersive interactions were considered by employing both a semi-empirical method at the PBE-D2 ${ }^{39}$ level and a parameter-free van der Waals density function (vdW-DF) method ${ }^{40}$. The optB86 $\mathrm{b}^{41}$ exchange was used to combine with the vdW correlation functional. The shape and volume of each superlattice were fully optimized and all atoms in it were allowed to relaxed until the residual force per atom was less than $0.01 \mathrm{eV} / \AA$. Layer alignment in superlattices was chosen with the energetically most favored configuration, as reported in Ref. 15. The SOC correction, which may sufficiently influence band energies $\left(0.16 \mathrm{eV}\right.$ and $0.4 \mathrm{eV}$ for $\mathrm{MoS}_{2}{ }^{11,42}$ and $\mathrm{WSe}_{2}{ }^{42,43}$ respectively), was included for electronic bandstructure calculations. It was found that the van der Waals functionals play a major role for geometry and energetics, but a minor role in electronic structures ${ }^{44}$. Therefore, in addition to PBE calculation, a hybrid functional, namely Heyd-Scuseria-Ernzerhof (HSE06) ${ }^{45}$, has been employed to address a known issue of underestimated bandgaps by PBE in the bandstructure calculation of the $1: 1$ superlattice.

The optical properties were obtained from PBE results, and the k-mesh was doubled in calculating dielectric functions. Excitonic contributions were not considered in our calculations.

The total number of bands considered was set to be twice that used in the totalenergy and bandstructure calculations.

1. Geim, A. K. \& Grogorieva, I. V. van der Waals heterostructures. Nature 499, 419-425 (2013).

2. Radisavljevic, B. et al. A. Single-layer $\mathrm{MoS}_{2}$ transistors. Nat. Nano. 6, 147-150 (2011).

3. Wang, Q. H. et al. Electronics and optoelectronics of two-dimensional transition metal dichalcogenides. Nat. Nano. 7, 699-712 (2012).

4. Xu, M. et al. Graphene-Like Two-Dimensional Materials. Chem. Rev. 113, 3766-3798 (2013).

5. Neto, A. H. C. \& Novoselov, K. New directions in science and technology: twodimensional crystals. Rep. Prog. Phys. 74, 082501 (2011)

6. Ji, Q. et al. Epitaxial Monolayer $\mathrm{MoS}_{2}$ on Mica with Novel Photoluminescence. Nano Lett. 13, 3870-3877 (2013).

7. Komsa, H.-P. \& Krasheninnikov, A. V. Electronic structures and optical properties of realistic transition metal dichalcogenide heterostructures from first principles. Phys. Rev. B 88, 085318 (2013). 
8. Britnell, L. et al. Strong Light-Matter Interactions in Heterostructures of Atomically Thin Films. Science 340, 1311-1314 (2013).

9. Bernardi, M., Palummo, M. \& Grossman, J. C. Extraordinary Sunlight Absorption and One Nanometer Thick Photovoltaics Using Two-Dimensional Monolayer Materials. Nano Lett. 13, 3664-3670 (2013).

10. Yun, W. S. et al. Thickness and strain effects on electronic structures of transition metal dichalcogenides: $2 \mathrm{H}-\mathrm{MX}_{2}$ semiconductors $(\mathrm{M}=\mathrm{Mo}, \mathrm{W} ; \mathrm{X}=\mathrm{S}, \mathrm{Se}, \mathrm{Te})$. Phys. Rev. B 85, 033305 (2012).

11. Mak, K. F. et al. Atomically Thin $\mathrm{MoS}_{2}$ : A New Direct-Gap Semiconductor. Phys. Rev. Lett. 105, 136805 (2010).

12. He, K. et al. Experimental Demonstration of Continuous Electronic Structure Tuning via Strain in Atomically Thin $\mathrm{MoS}_{2}$. Nano Lett. 13, 2931-2936 (2013).

13. Scalise, E. et al. Strain-Induced Semiconductor to Metal Transition in the TwoDimensional Honeycomb Structure of $\mathrm{MoS}_{2}$. Nano Res. 5, 43-48 (2012).

14. Peelaers, H. et al. Van de Walle, C. G. Effects of strain on band structure and effective masses in $\mathrm{MoS}_{2}$. Phys. Rev. B 86, 241401(R) (2012).

15. Terrones, H., López-Urías, F. \& Terrones, M. Novel hetero-layered materials with tunable direct bandgaps by sandwiching different metal disulfides and diselenides. Sci. Rep. 3, 1549 (2013)

16. Kośmider, K. \& Fernández-Rossier, J. Electronic properties of the MoS2-WS2 heterojunction. Phys. Rev. B 87, 075451 (2013).

17. Kobayashi, K. \& Yamauchi, J. Electronic structure and scanning-tunnelingmicroscopy image of molybdenum dichalcogenide surfaces. Phys. Rev. B 51, 17085-17095 (1995)

18. Lebégue, S. \& Eriksson, O. Electronic structure of two-dimensional crystals from ab initio theory. Phys. Rev. B 79, 115409 (2009).

19. Yousefi, G. H. Optical properties of mixed transition metal dichalcogenide crystals. Mater. Lett. 9, 38-40 (1989).

20. Traving, M. et al. Electronic structure of $\mathrm{WSe}_{2}$ : A combined photoemission and inverse photoemission study. Phys. Rev. B 55, 10392-10399 (1997).

21. Jin, W. et al. Direct Measurement of the Thickness-Dependent Electronic Band Structure of $\mathrm{MoS}_{2}$ Using Angle-Resolved Photoemission Spectroscopy. Phys. Rev. Lett. 111, 106801 (2013).

22. Sahin, H. et al. Anomalous Raman spectra and thickness-dependent electronic properties of $\mathrm{WSe}_{2}$. Phys. Rev. B 87, 165409 (2013).

23. Qiao, J. et al. High-mobility transport anisotropy and linear dichroism in fewlayer black phosphorus. Nat. Commun. 5, 4475 (2014).

24. Feng, J., Qian, X., Huang, C. \& Li, J. Strain-engineered artificial atom as a broadspectrum solar energy funnel. Nat. Photon. 6, 866-872 (2012).

25. Conley, H. J. et al. Bandgap Engineering of Strained Monolayer and Bilayer $\mathrm{MoS}_{2}$. Nano Lett. 13, 3626-3630 (2013).

26. Desai, S. B. et al. Strain-Induced Indirect to direct Bandgap Transition in Multilayer WSe $\mathrm{W}_{2}$. Nano Lett. 14, 4592-4597 (2014).

27. Krukau, A. V. et al. Influence of the exchange screening parameter on the performance of screened hybrid functionals. J. Chem. Phys. 125, 224106 (2006).

28. Hong, X. et al. Ultrafast Charge Transfer in Atomically Thin MoS2/WS2 Heterostructures. Nat. Nano. 9, 682 (2014).

29. $\mathrm{Wu}, \mathrm{S}$. et al. Electrical tuning of valley magnetic moment through symmetry control in bilayer $\mathrm{MoS}_{2}$. Nat. Phys. 9, 149-153 (2013).

30. Cao, T. et al. Valley-selective circular dichroism of monolayer molybdenum disulphide. Nat. Commun. 3, 887 (2012).

31. Xiao, D. et al. Coupled Spin and Valley Physics in Monolayers of $\mathrm{MoS}_{2}$ and Other Group-VI Dichalcogenides. Phys. Rev. Lett. 108, 196802 (2012).

32. Heideman, C. L. et al. Designed Synthesis, Structure, and Properties of a Family of Ferecrystalline Compounds $\left[(\mathrm{PbSe})_{1.00}\right]_{m}\left(\mathrm{MoSe}_{2}\right)_{n}$. J. Am. Chem. Soc. 135, 11055-11062 (2013).

33. Sun, L. et al. Spin-Orbit Splitting in Single-Layer $\mathrm{MoS}_{2}$ Revealed by Triply Resonant Raman Scattering. Phys. Rev. Lett. 111, 126801 (2013).

34. Perdew, J. P., Burke, K. \& Ernzerhof, M. Generalized Gradient Approximation Made Simple. Phys. Rev. Lett. 77, 3865-3868 (1996).

35. Blöchl, P. E. Projector augmented-wave method. Phys. Rev. B 50, 17953-17979 (1994)
36. Kresse, G. \& Joubert, D. From ultrasoft pseudopotentials to the projector augmented-wave method. Phys. Rev. B 59, 1758-1775 (1999).

37. Kresse, G. \& Furthmüller, J. Efficient iterative schemes for $a b$ initio total-energy calculations using a plane-wave basis set. Phys. Rev. B 54, 11169-11186 (1996).

38. Kresse, G. \& Furthmüller, J. Efficiency of ab-initio total energy calculations for metals and semiconductors using a plane-wave basis set. Comput. Mat. Sci. 6, 15-50 (1996).

39. Grimme, S. Semiempirical GGA-Type Density Functional Constructed with a Long-Range Dispersion Correction. J. Comput. Chem. 27, 1787-1799 (2006).

40. Román-Pérez, G. \& Soler, J. M. Efficient Implementation of a van der Waals Density Functional: Application to Double-Wall Carbon Nanotubes. Phys. Rev. Lett. 103, 096102 (2009).

41. Klimes, J., Bowler, D. R. \& Michaelides, A. Van der Waals density functionals applied to solids. Phys. Rev. B 83, 195131 (2011).

42. Zhu, Z. Y., Cheng, Y. C. \& Schwingenschlögl, U. Giant spin-orbit-induced spin splitting in two-dimensional transition-metal dichalcogenide semiconductors. Phys. Rev. B 84, 153402 (2011).

43. Zhao, W. et al. Evolution of Electronic Structure in Atomically Thin Sheets of $\mathrm{WS}_{2}$ and $\mathrm{WSe}_{2}$. ACS Nano 7, 791 (2013).

44. Hu, Z.-X., Lan, H. P. \& Ji, W. Role of the dispersion force in modeling the interfacial properties of molecule-metal interfaces: adsorption of thiophene on copper surfaces. Sci. Rep. 4, 5036 (2014)

45. Heyd, J., Scuseria, G. E. \& Ernzerhof, M. Erratum: Hybrid functionals based on a screened Coulomb potential. J. Chem. Phys. 124, 219906 (2006).

\section{Acknowledgments}

This work was financially supported by the National Natural Science Foundation of China (NSFC), Grant Nos. 11104191, 11047104, 11004244, 11274380 and 91433103, the Beijing Natural Science Foundation (BNSF), Grant No. 2112019, and the Basic Research Funds in Renmin University of China from the Central Government (Grant No. 12XNLJ03), the Ministry of Science and Technology (MOST) of China (Grant No. 2012CB932704). W.J. was supported by the Program for New Century Excellent Talents in University. Y.Z. and J.W. was supported by the Hong Kong University Grant Council (Contract No. AoE/P-04/ 08) of the Government of HKSAR. Calculations were performed at the Physics Lab of High-Performance Computing of Renmin University of China, Shanghai Supercomputer Center, and High-Performance Computing Center at Sichuan Normal University. We thank Profs. Fuchun Zhang and Qingfeng Sun for fruitful discussions.

\section{Author contributions}

Y.Z. and W.J. conceived the whole research and perform all calculations. F.Y. contributed to the SOC calculation of bulk TMDCs. J.W. and H.G. participate the explanation of results. Y.Z., J.W., H.G. and W.J. wrote the manuscript.

\section{Additional information}

Supplementary information accompanies this paper at http://www.nature.com/ scientificreports

Competing financial interests: The authors declare no competing financial interests.

How to cite this article: Zhao, Y.-H., Yang, F., Wang, J., Guo, H. \& Ji, W. Continuously tunable electronic structure of transition metal dichalcogenides superlattices. Sci. Rep. 5, 8356; DOI:10.1038/srep08356 (2015).

This work is licensed under a Creative Commons Attribution 4.0 International License. The images or other third party material in this article are included in the article's Creative Commons license, unless indicated otherwise in the credit line; if the material is not included under the Creative Commons license, users will need to obtain permission from the license holder in order to reproduce the material. To view a copy of this license, visit http://creativecommons.org/licenses/by/4.0/ 